\title{
Guest editorial: Special section: Software quality and maintainability
}

\author{
Yiannis Kanellopoulos • Yijun Yu
}

Published online: 14 February 2015

(C) Springer Science+Business Media New York 2015

Software plays a crucial role in modern societies. Not only do people rely on it for their daily operations or business, but also for their lives as well. For this reason, correct and consistent behavior of software systems is a fundamental and critical part of end-user expectations. Additionally, businesses require cost-effective production, maintenance, and operation of their systems. Thus, the demand for high-quality and maintainable software is increasing, setting it as a differentiator for the success or failure of a software product, as well as a dominating factor for the commercial success of companies.

The main questions that arise concern how quality is measured and communicated to stakeholders. What, where, and when we assess and assure quality are also open issues. Many views have been expressed about software quality attributes including maintainability, security, evolvability, portability, robustness, reliability, usability, and efficiency. Some of these have been formulated in standards such as ISO/IEC 25010 (SQuaRE) and CMMI. However, the debate about quality and maintainability between software producers, vendors, and users continues, while organizations need the ability to evaluate the software systems they use or develop from multiple viewpoints. So, is "software quality in the eye of the beholder"?

This special section on Software Quality and Maintainability presents original work and relates experiences on issues pertaining to software quality and maintainability. Moreover, the theme of this special section opens discussion of how to investigate quality issues in the maintenance of software systems.

Out of 15 full-paper submissions for this issue, four papers were selected. The accepted papers address a range of topics related to software quality, including code refactoring, API

Y. Kanellopoulos $(\bowtie)$

Software Improvement Group, Amstelplein 1, 1096 HA Amsterdam, The Netherlands e-mail: y.kanellopoulos@sig.eu

Y. Yu

Department of Computing and Communications, The Open University, Milton Keynes, MK7 6AA Buckinghamshire, UK

e-mail: y.yu@open.ac.uk 
usage, and test coverage, as well as how to automate instrumentation tools. Specifically, the paper by Chaikalis et al. reports on the investigation of evolution and refactoring on the feature scattering in several open-source software projects. The paper by Serebrenik et al. presents an empirical evaluation of the API usages of the Eclipse IDE itself. Zhang et al. present a declarative approach to instrumentation of Java code which has found application in several code quality analysis tools. Fawaz et al. present property-based criteria for testing coverage of software quality. We hope this selection of archived articles can push the state-of-the-art of software quality and maintenance forward. 